\title{
A Lie Algebraic Approach to Design of Stable Feedback Control Systems with Varying Sampling Rate
}

\author{
Flavia E. Felicioni*. Sergio J. Junco** \\ * Departamento de Electrónica - FCEIyA - UNR Universidad Nacional de Rosario, \\ Rosario-Argentina, CONICET (e-mail:flaviaf@,fceia.unr.edu.ar) \\ ** Departamento de Electrónica - FCEIyA - UNR Universidad Nacional de Rosario, \\ Rosario-Argentina, (e-mail: sjunco@fceia.unr.edu.ar)
}

\begin{abstract}
This paper addresses the design of a controller family that, given a continuous-time linear plant sampled at a varying rate, asymptotically stabilizes the closed loop. Under the assumption of having a finite and known set of allowable sampling intervals, the problem is formulated as that of stabilizing a discrete-time switched system (DTSS). The solution approach consists in choosing the controller parameters in order for the Lie algebra generated by the closed-loop DTSS-matrices to be solvable, as this property guarantees the existence of a common Lyapunov function for the control system. The results can be applied to design digital controllers sharing resources, as it is the case of networked control systems, where the need of adapting the rate of task scheduling may originate significant sampling time variations.
\end{abstract}

\section{INTRODUCTION}

Point to point communication and dedicated central processing unit (CPU) traditionally used in control systems implementations are being replaced in many applications by distributed communication (via a common network) and/or shared CPUs (at each node or CPU there are several tasks related with the control of different systems).

The use of common shared resources reduces costs and provides better resource's throughput, benefits that are frequently accompanied by noticeable increments in the amount of information exchanged between the system nodes. As a result, the real-time deadlines of measurement and actuation tasks may be violated due to execution delays, which can lead to performance degradation or even instability of a control system.

In order to mitigate these problems, several measures to achieve an efficient use of available resources have been suggested, rate adaptation of task scheduling among them. For a shared CPU, a feedback scheduler that adjusts the sampling rates of the control loops to guarantee schedulability in different resource conditions was used in (Eker et al., 2000) and (Cervin et al, 2002). In a context of networked control systems, (Antunes et al, 2007), (Velazco et al., 2004), and (Felicioni and Junco, 2007), present techniques to adapt the communication rate of the distributed controllers to the available network bandwidth.

Rate adaptation introduces the need to design control laws adapted to these variations and aimed at reducing the performance degradation induced by a varying sampling rate if invariant (non rate-adaptive) controllers were used and, simultaneously, ensuring closed-loop stability.

In order to adapt control laws to rate variations, an optimal design of state-feedback linear quadratic controllers (LQ) was proposed in (Cervin et al., 2002). But, as shown in (Schinkel et al., 2002), control systems designed with this optimal-LQ technique, may suffer from instability under certain switching sequences. Due to this undesirable result, (Schinkel et al., 2002) adopts a linear matrix inequalities (LMI) approach to design stable optimal controllers. However, their numerical solutions can be very conservative (see section 4.2).

As a given control system undergoing sampling rate variation can be thought of as a concatenation of systems in time, it can be modeled as a Discrete-Time Switched System (DTSS). This is the mathematical framework exploited in this paper for the control system design problem.

A review of available results to study asymptotic stability of switched systems was presented in (Liberzon and Morse, 1999). In particular, it was established that if a family of systems that constitutes a switched system has a Common Lyapunov Function (CLF), then, asymptotic stability is guaranteed for any switching sequence. Furthermore, global uniform (for all switching signals) exponential stability was proved for a continuous-time switched system described by a compact family of linear systems, under the condition that each matrix in the family is stable and the Lie algebra associated with the family is solvable. This result was demonstrated connecting the Lie algebra solvability with the existence of a Quadratic CLF. A discrete-time counterpart of this result was presented in (Blondel et al., 2004) and (Theys, 2005). 
Here, we propose to use Blondel-Theys's result to solve the following problem: given a linear plant sampled at varying rate, design a controller family that asymptotically stabilizes the closed-loop for a given sampling policy.

\section{PROBLEM FORMULATION AND BACKGROUND}

This section first formalizes the description of a control system with varying sampling rate as a DTSS. It proceeds with the closer examination of related results and mathematical tools used to formulate and solve the problem.

\subsection{Switched Linear Systems}

Definition (Hespanha and Morse,1999) A Continuous-Time Switched Linear System is defined by a parameterised family of realizations $P=\left\{\left(\mathrm{A}_{\mathrm{i}}, \mathrm{B}_{\mathrm{i}}, \mathrm{C}_{\mathrm{i}}, \mathrm{D}_{\mathrm{i}}\right): \mathrm{i} \in \underline{p}=\{1,2, \ldots, p\}\right\}$, together with a family of piecewise constant switching signals $\mathrm{S}:=\{\sigma:[0, \infty) \rightarrow p\}$, and the (input-state-output) dynamics (1).

$$
\begin{aligned}
& \dot{x}(t)=A_{\sigma(t)} x(t)+B_{\sigma(t)} u(t) \\
& y(t)=C_{\sigma(t)} x(t)+D_{\sigma(t)} u(t)
\end{aligned}
$$

Analogously, a Discrete-Time Switched Linear System with the discrete time base $\left\{\mathrm{t}_{\mathrm{k}}: k \in \mathbb{N}_{0}\right\}$ has the following form:

$$
\begin{aligned}
& x_{k+1}=A_{\sigma(k)} x_{k}+B_{\sigma(k)} u_{k} \\
& y_{k}=C_{\sigma(k)} x_{k}+D_{\sigma(k)} u_{k}
\end{aligned}
$$

With $u_{k}=u\left(t_{k}\right), x_{k}=x\left(t_{k}\right)$, and $y_{k}=y\left(t_{k}\right)$.

Due to the switching of the matrices inside the family $P$, both systems, (1) and (2), are time-varying.

\subsection{Varying-rate Sampled-data Control Systems}

The discretization with a sampling time $h_{i}=t_{k+1}-t_{k}$ of the open-loop continuous-time plant described by the linear model (3) yields the discrete-time linear system (4), whose matrices are given in (5), if a zero-order hold is considered.

$$
\begin{gathered}
\dot{x}=A x+B u \\
y=C x+D u \\
x_{k+1}=\Phi\left(h_{i}\right) x_{k}+\Gamma\left(h_{i}\right) u_{k} \\
y_{k}=C_{d}\left(h_{i}\right) x_{k}+D_{d}\left(h_{i}\right) u_{k} \\
\Phi\left(h_{i}\right)=e^{A h_{i}} \quad C_{d}\left(h_{i}\right)=C \\
\Gamma\left(h_{i}\right)=\int_{0}^{h_{i}} e^{A s} B d s \quad D_{d}\left(h_{i}\right)=D
\end{gathered}
$$

For varying sampling time $h_{i}$, system (4) can be modeled as (2), with $\sigma(k)=i \in \underline{p}$, which yields the equivalence of matrices (6).

$$
\begin{aligned}
A_{i} & =\Phi\left(h_{i}\right) \\
B_{i} & =\Gamma\left(h_{i}\right) \\
C_{i} & =C\left(h_{i}\right) \\
D_{i} & =D\left(h_{i}\right)
\end{aligned}
$$

A Closed-Loop Discrete-Time System results from the interconnection of the sampled plant with the linear discretetime dynamic controller (7). Note that the controller parameters adapt themselves to the sampling period.

$$
\begin{aligned}
& v_{k+1}=A_{C, i} v_{k}+B_{C, i} C_{d}\left(h_{i}\right) x_{k} \\
& u_{k}=C_{C, i} v_{k}+D_{C, i} C_{d}\left(h_{i}\right) x_{k}
\end{aligned}
$$

Defining the augmented state $\left(\begin{array}{lll}x_{k} & v_{k}\end{array}\right)^{T}$, then the closed-loop dynamics is described by (8)

$$
\begin{aligned}
\left(\begin{array}{c}
x_{k+1} \\
v_{k+1}
\end{array}\right) & =\left(\begin{array}{cc}
\Phi\left(h_{i}\right)+\Gamma\left(h_{i}\right) \cdot D_{C, i} \cdot C\left(h_{i}\right) & \Gamma\left(h_{i}\right) \cdot C_{C, i} \\
B_{C, i} \cdot C\left(h_{i}\right) & A_{C, i}
\end{array}\right) \cdot\left(\begin{array}{c}
x_{k} \\
v_{k}
\end{array}\right) \\
& =\Phi_{i}^{C L} \cdot\left(\begin{array}{c}
x_{k} \\
v_{k}
\end{array}\right)
\end{aligned}
$$

Particularly, for a static state feedback controller $u_{k}=L_{i} x_{k}$, the closed-loop matrix of equation (8) reduces to

$$
\Phi_{i}^{C L}=\Phi\left(h_{i}\right)+\Gamma\left(h_{i}\right) L_{i}=\Phi_{i}+\Gamma_{i} L_{i}
$$

\subsection{Asymptotic Stability in Closed-loop.}

The problem to solve in this paper is the following:

Given the family describing the open-loop systems (4), associated with a finite number of sampling periods $\left\{h_{i}, i \in\right.$ $\underline{p}\}$, find a family of controllers $\left(A_{C, i}, B_{C, i}, C_{C, i}, D_{C, i} ; i \in \underline{p}\right)$ such that the DTSS closed-loop system (9) is asymptotically stable.

Variations of this controller design problem have been approached in (Sala, 2005) and (Schinkel et al., 2002) adopting a LMI framework to synthesize optimal controllers that guarantee closed-loop stability under any sampling sequence. More specifically, Sala solves the problem with a unique common controller, while Schinkel obtains a family of controllers associated to the sampling rates.

The solution provided by this work consists of a family of stabilizing controllers with the property that the resulting closed-loop descriptions have a Quadratic CLF (QCLF). It is assumed that the switching signal (which generates the varying parameter $h_{i}$ in our system (8)) is determined by a scheduler policy yielding a finite number of known sampling periods.

As reported in (Liberzon and Morse, 1999), the existence of a QCLF for a family of (autonomous) systems guarantees asymptotic stability for arbitrary switching signals. Blondel and Theys, approaching DTSS in a similar way as (Liberzon and Morse, 1999) did for continuous-time systems, proved that if the Lie algebra associated to a family of stable matrices is solvable, then, there exists a QCLF. This result is quoted in the theorem below.

Theorem [Blondel] [Theys]. Let $P$ be the family of matrices $\left\{M_{1}, M_{2} \cdots, M_{p}\right\}$ and consider the switched linear system $x_{k+1}=M_{i} x_{k}, M_{i} \in P$. If all matrices in $P$ have spectral radius $\rho\left(M_{i}\right)$ less than 1 (largest modulus of their 
eigenvalues) and the Lie algebra associated to $P$ is solvable, then the system has a QCLF.

An equivalent result was first established in (Gurvits, 1995) for the particular case where each $M_{i}$ in the family takes the form $e^{A i}$.

In this paper, the matrices $M_{i}$ are the closed loop matrices $\Phi_{i}^{C L}$, i.e., the approach to solve the stability problem consists in imposing the conditions of the precedent theorem to the family of matrices $P=\left\{\Phi_{i}^{C L} ; i \in \underline{p}\right\}(8)$, in order to design the family of controllers $\left(A_{C, i}, B_{C, i}, C_{C, i}, D_{C, i} ; i \in \underline{p}\right)$ looked for.

\subsection{Mathematical Background}

The matrix Lie algebra $\mathrm{g}:=\left\{M_{i}: i \in \underline{p}\right\}$ generated by the family of square matrices $P=\left\{M_{1}, M_{2} \cdots, M_{p}\right\}$ wrt the standard Lie bracket $\left[M_{v}, M_{\gamma}\right]=M_{v} M_{\gamma}-M_{\gamma} M_{v}$, is the linear space spanned by iterated Lie brackets of matrices of the family (Liberzon et al.,1999), (Theys, 2005).

For the Lie algebra $g$, the sequence $\mathrm{g}^{(\mathrm{m})}$ is defined as $\mathrm{g}^{(0)}:=\mathrm{g}$, $\mathrm{g}^{(\mathrm{m}+1)}:=\left[\mathrm{g}^{(\mathrm{m})}, \mathrm{g}^{(\mathrm{m})}\right] \subset \mathrm{g}^{(\mathrm{m})}$.

If $\mathrm{g}^{(\mathrm{m})}=0$ for some $\mathrm{m}$, then $\mathrm{g}$ is said to be solvable.

Given the Lie algebra $\mathrm{g}$, the sequence $\mathrm{g}^{\mathrm{m}}$ is defined as $\mathrm{g}^{0}:=\mathrm{g}$, $\mathrm{g}^{\mathrm{m}+1}:=\left[\mathrm{g}, \mathrm{g}^{\mathrm{m}}\right] \subset \mathrm{g}^{\mathrm{m}}$.

If $\mathrm{g}^{\mathrm{m}}=0$ for some $\mathrm{m}$, then $\mathrm{g}$ is called nilpotent.

The nilpotency of a Lie algebra is a sufficient condition for its solvability.

Also, the Lie algebra $\mathrm{g}:=\left\{M_{i}: i \in \underline{p}\right\}$ is solvable if and only if the Lie algebra $\widetilde{\mathrm{g}}:=\left\{\tilde{M}_{i}: i \in \underline{p}\right\}$ is solvable, where $\tilde{M}_{i}=R^{-1} M_{i} R$, with $R$ the matrix of a similarity transformation.

\section{CONTROLLER DESIGN}

As already pointed-out, we propose here to choose the controller parameters in order for the Lie algebra generated by the closed-loop DTSS-matrices (8) to be solvable. More explicitly, as the closed-loop model (8) is associated to each sampling time, a family of closed-loop matrices is recovered, each of them being parameterized by the corresponding controller set of parameters, which have to be determined in such a way that the sequence $\mathrm{g}^{(\mathrm{m})}$ generated by the closedloop family is forced to be zero for some $\mathrm{m}$.

Three cases are presented in the sequel, and the conditions to obtain solvable algebras associated with a family of DTSS matrices are given. These methods are illustrated by some examples in the next section.

Case 1: If $\mathrm{g}^{(1)}=0$, the algebra is solvable, the matrices are pairwise commutative (abelian case), i.e. all the brackets between matrices in the set $P=\left\{\Phi_{1}^{C L}, \Phi_{2}^{C L}, \cdots, \Phi_{P}^{C L}\right\}$ are zero. This is an application of a particular case of Blondel's Theorem, which was first proved in (Kumpati et al., 1994).
For each pair of matrices in the family, this condition requires that $\left[\Phi_{1}^{C L}, \Phi_{2}^{C L}\right]=\Phi_{1}^{C L} \Phi_{2}^{C L}-\Phi_{2}^{C L} \Phi_{1}^{C L}=0$. To do that, the controller parameters must to satisfy the following design matrix equation

$$
\Phi_{1}^{C L} \Phi_{2}^{C L}=\Phi_{2}^{C L} \Phi_{1}^{C L}
$$

For the controller structure in (9), due to $\Phi_{1} \Phi_{2}=e^{A h_{1}} e^{A h_{2}}=\Phi_{2} \Phi_{1},(10)$ reduces to

$$
\Phi_{1} \Gamma_{2} L_{2}+\Gamma_{1} L_{1} \Gamma_{2} L_{2}=\Phi_{2} \Gamma_{1} L_{1}+\Gamma_{2} L_{2} \Gamma_{1} L_{1}
$$

For a closed-loop matrix of order $n \times n$ the matrix equation (11) implies $n^{2}$ equations to satisfy. The possibility of satisfying these constraints depends on the number of openloop inputs and outputs and the chosen controller structure.

Case 2: The algebra generated by a family of upper (or lower) triangular matrices is solvable, with $\mathrm{m}=\mathrm{n}+1$. As (8) typically does not have a triangular structure, this proposition requires to put (10), in a triangular form by using a similarity transformation common to all.

From digital control theory, the exponential map of the openloop system $A=R T R^{-1}$ is

$$
e^{A t}=e^{R T R^{-1} t}=R e^{T t} R^{-1}
$$

where $R$ is a similarity transformation. If it is a Jordan decomposition, $T$ has an upper (or lower) triangular form (or a diagonal one).

Applying this transformation to the input part in (6)

$$
\Gamma(t)=\int_{0}^{t} e^{A x} B d s=\int_{0}^{t} R e^{T x} R^{-1} B d s=R\left(\int_{0}^{t} e^{T x} d s\right) R^{-1} B=R G_{t} R^{-1} B
$$

Here, we consider a state-feedback controller, then by using (13) and (4), (10) becomes

$$
x_{k+1}=R\left(e^{T t}+G_{t}\left(R^{-1} B L_{t} R\right)\right) R^{-1} x_{k}
$$

If $e^{T t}+G_{t}\left(R^{-1} B L_{t} R\right)$ has an upper (or lower) triangular form for each sampling time $t=h_{i}$ in the set $\left\{h_{i}, i \in \underline{p}\right\}$, then the algebra associated to closed-loop family results solvable.

As for a Jordan decomposition $e^{T t}$ has an upper (or lower) triangular form, we propose to design state-feedback controller $L_{t}$ in such a way that each matrix $G_{t}\left(R^{-1} B L_{t} R\right)$ has an upper (or lower) triangular form.

Depending on the open-loop structure, the satisfaction of this condition requires to choose some controller parameters equal to zero. Thus, in several cases, some degrees of freedom for the election of gains of the state-feedback controllers are lost.

Case 3: Here, we analyse the solvability of the algebra generated by a pair of matrices of order 2 .

In order to design the pair of controllers, we compute some brackets and derive two equations that must be satisfied to obtain a solvable algebra. 
Consider closed-loop matrices $M_{1}$ and $M_{2}$, then the sum of diagonal elements of first bracket $\left[M_{1}, M_{2}\right]=M_{3}$ is zero, i.e. $M_{3(1,1)}=-M_{3(2,2)}$ (where $M_{i(k, j)}$ refers to the element of column $k$ and row $j$ from matrix $M_{i}$ ). From the computation of next brackets, $M_{3}=\left[M_{1}, M_{2}\right], \quad M_{4}=\left[M_{1}, M_{3}\right], \quad M_{5}=\left[M_{2}, M_{3}\right]$, $M_{6}=\left[M_{3}, M_{4}\right], M_{7}=\left[M_{3}, M_{5}\right], M_{8}=\left[M_{6}, M_{7}\right]$, and so on, it is possible to verify for all $i$ in the set, the same diagonal elements condition, i.e. $M_{i(1,1)}=-M_{i(2,2)}$ and $M_{i(1,1)}=-M_{i(2,2)}$. The generated algebra for this pair is $\mathrm{g}^{(0)}=\operatorname{span}\left\{M_{1}, M_{2}, M_{3}, M_{4}\right.$, $\left.M_{5}, M_{6}, M_{7}, M_{8}, \ldots\right\}, \mathrm{g}^{(1)}=\operatorname{span}\left\{M_{3}, M_{4}, M_{5}, M_{6}, M_{7}, M_{8}, \ldots\right\}$, $\mathrm{g}^{(2)}=\operatorname{span}\left\{M_{4}, M_{5}, M_{6}, M_{7}, M_{8}, \ldots\right\}, \mathrm{g}^{(3)}=\operatorname{span}\left\{M_{6}, M_{7}, M_{8}, \ldots\right\}$, $\mathrm{g}^{(4)}=\left\{M_{8}, \ldots\right\}$ and so on.

Consider a pair of indices $k$ and $j$ such that $k \neq j, r \leq\{k, j\} \leq r+2$, for some $r$, and the elements $M_{k(1,1)}, M_{k(1,2)}, M_{k(2,1)}, M_{k(2,2) \text {, }}$ $M_{j(1,1)}, M_{j(1,2)}, M_{j(2,1)}, M_{j(2,2)}$ are non-zero. To obtain that the next common bracket $\left[M_{k}, M_{j}\right]$ gives a $0_{2 * 2}$ we must to satisfy both following equations:

$$
M_{k(1,2)}=\frac{M_{k(1,1)} M_{j(1,2)}}{M_{j(1,1)}} M_{k(2,1)}=\frac{M_{k(1,1)} M_{j(2,1)}}{M_{j(1,1)}}
$$

The iterated bracket computation produces non-linear combinations of the controller parameters in the elements in equation (15) (typically they are linear in matrices $M_{l}, M_{2}$ ). Thus, the solution of these equations becomes more complicated when the value of $\mathrm{m}$ is increased. Clearly, nonlinear methods must be required to solve this problem.

\section{EXAMPLES}

This section shows some examples to illustrate the methods mentioned in the last section to satisfy the Lie algebra conditions in each of the three cases.

Finding the solutions requires solving a set of non-linear equations. That can be done in symbolic or numerical forms. For example, in the first case by using the symbolic method solve from MAPLE, or in the latter case by using the numerical methods $f$ solve from MATLAB or MAPLE.

\subsection{An Introductory Example}

In the first order system taken from (Cervin et al., 2002), without noise, controlled by state-feedback, the closed-loop equation for a finite number of sampling periods $\left\{h_{i}, i \in \underline{p}\right\}$ is $\Phi_{k, i}^{C L}=e^{a h_{i}}+\frac{1}{a}\left(e^{a h_{i}}-1\right) l_{i}$, i.e. it is a scalar value. Therefore, the algebra generated by family $\left\{\Phi_{1}^{C L}, \Phi_{2}^{C L}, \cdots, \Phi_{P}^{C L}\right\}$ is always solvable $\left(\mathrm{g}^{(1)}=0\right)$. Furthermore, if $\left|e^{a h_{i}}+\frac{1}{a}\left(e^{a h_{i}}-1\right) l_{i}\right|<1$ for all $i$, both hypothesis of Theorem in subsection 2.3 are satisfied, implying the asymptotic stability under any sampling sequence.

The closed-loop for a second sampling time $h_{2}=\alpha h_{1}$, being $\alpha$ an integer, is $\Phi_{2}^{C L}=e^{a \alpha h_{1}}+\frac{1}{a}\left(e^{a \alpha h_{1}}-1\right) l_{2}$. Due to the simple structure of this example, it is possible to obtain that $\Phi_{2}^{C L}=\left(\Phi_{1}^{C L}\right)^{\alpha}$ in time $t=\alpha h_{1}+t_{0}$.
For example if $\alpha=2$, the satisfaction of the mentioned equation requires to adopt $l_{2}=\frac{l_{1}\left(2 a e^{a 2 h_{1}}-l_{1}+e^{a h_{1}} l_{1}\right)}{a\left(1+e^{a 2 h_{1}}\right)}$. With this adaptation of the control parameter, the plant reaches the same final state value in one step of sampling time $h_{2}$ or in two steps of sampling time $h_{l}$. Then, this election of $l_{2}$ allows us to compensate the augmented sampling interval with no degradation in terms of plant state response.

\subsection{Numerical Example}

Here, we solve a numerical problem as an example of Case 3. Due to the numerical distortion introduced by the discretization (5), it is necessary to consider the number precision as large as possible in order to obtain a good numerical solution.

For the second order plant taken from (Schinkel et al., 2002)

$$
\dot{x}=\left[\begin{array}{cc}
0 & 1 \\
-10000 & -0.1
\end{array}\right] x+\left[\begin{array}{l}
0 \\
1
\end{array}\right] u
$$

with two sampling times $(P=2), h_{l}=0.002$ and $\varepsilon=15$ (see note), the family of closed-loop matrices (9) is

$$
\begin{gathered}
\left\{\Phi_{1}^{C L}=\left[\begin{array}{cc}
0.9801 & 0.0020 \\
-19.8649+0.0020 l_{l, 1} & 0.9799+0.0020 l_{l, 2}
\end{array}\right],\right. \\
\left.\Phi_{\varepsilon}^{C L}=\left[\begin{array}{cc}
-0.9884+0.0002 l_{2,1} & 0.0014+0.0002 l_{2,2} \\
-14.0909+0.0014 l_{2,1} & -0.9886+0.0014 l_{2,2}
\end{array}\right]\right\}
\end{gathered}
$$

To obtain the controller parameters for this family, we first compute the seven first Lie brackets as exposed in Case 3. Assuming $k=7$ and $j=6$, we derive the non-linear equations (15) to obtain $M_{8}=\left[M_{6}, M_{7}\right]=0_{2 * 2}$. Then, the algebra $\mathrm{g}$ associated with this pair $\left\{\Phi_{C l, 1}, \Phi_{C l, \varepsilon}\right\}$ is solvable being $m=4$.

The parameters that satisfy equations (15) were obtained by further calculations of fsolve command, initialized with a starting guess (parameters designed with LQR in (Schinkel et al., 2002), see also the Remark below).

$$
\begin{aligned}
& L_{1}=\left[\begin{array}{ll}
177.820193769818 & -19.683275734678
\end{array}\right] \\
& L_{\varepsilon}=\left[\begin{array}{ll}
140.481223090893 & -0.113548333882
\end{array}\right]
\end{aligned}
$$

The spectral values for closed loop matrices are $\rho\left(\Phi_{1}^{C L}\right)=$ $0.97997188<1$ and $\rho\left(\Phi_{\varepsilon}^{C L}\right)=0.984352978<1$ and $\mathrm{g}^{(4)}=0$, then the hypothesis of Theorem in Section 2 are satisfied and it guarantees asymptotic stability for arbitrary switching.

The difference between the final value of $L_{l}$ and the starting one, $L_{1,0}=[195.401-19.4121]$ designed with LQR, is rather small compared with the difference between $L_{1,0}$ and $L_{l, f}=[0.5784-0.0507]$ obtained via $L M I$ by Schinkel et al..

Remark: From digital control theory the sampling time must be chosen under the limit established by Shannon theorem $\left(h<\pi / \omega_{0}\right)$ In (Schinkel et al., 2002) that limit is not respected by the second sampling time, i.e. 0.094. For that reason, we select $h_{2}=0.03(\varepsilon=15)(h<\pi / 99.999)$.

The controller parameters for this sampling time $h_{2}$, L2 $=\left[\begin{array}{lll}-130.29664515561 & 1.123104069925\end{array}\right]$, were designed as 
in (Schinkel et al., 2002). For example, if the switching signal is $\sigma=\{1,2\}$, the dynamics switches between $\Phi_{1}^{C L}$ and $\Phi_{\varepsilon}^{C L}$ and always in that sequence, the augmented sampling time system is a periodic one, described by $\Phi_{1+e}^{C L}=\Phi_{1}^{C L} * \Phi_{\varepsilon}^{C L}$. The spectral radius is $\rho\left(\Phi_{1+e}^{C L}\right)>1$ shows that, even each matrix is stable, a sequence of them it is not.

\subsection{Symbolic Example-Commuting matrices}

Here, we solve a symbolic problem in the frame of Case 1 for the linear second order model of the cart system of Fig 1.

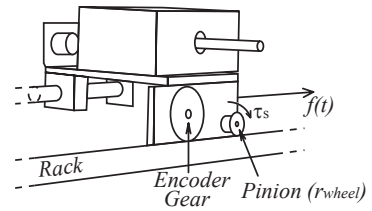

Figure 1: Cart System

The closed-loop system with a state-feedback controller, for $h_{i}=\alpha h_{1}$ with $\alpha \geq 1$, is

$$
\left[\begin{array}{cc}
\frac{k_{2}}{k_{1}}\left(\alpha h_{1}-\frac{\left(1-e^{-k_{1} \alpha h_{1}}\right)}{k_{1}}\right) l_{\alpha, 1} & \frac{1-e^{-k_{1} \alpha h_{1}}}{k_{1}}-\frac{k_{2}}{k_{1}}\left(\alpha h_{1}-\frac{\left(1-e^{-k_{1} \alpha h_{1}}\right)}{k_{1}}\right) l_{\alpha, 1} \\
\frac{k_{2}}{k_{1}}\left(1-e^{-k_{1} \alpha h_{1}}\right) l_{\alpha, 2} & e^{-k_{1} \alpha h_{1}}-\frac{k_{2}}{k_{1}}\left(1-e^{-k_{1} \alpha h_{1}}\right) l_{\alpha, 2}
\end{array}\right]
$$

Symbolic solution of (11) for the controller $L_{\alpha}$ is presented in Appendix I (equations (17) and (18)). This controller can be calculated as a non-linear function depending on plant parameters $k_{l}$ and $k_{2}$, the sampling times $h_{l}$ and $\alpha h_{l}$, and the controller parameters $L_{l}$ designed via traditional methods (pole-placement, LQR,..).

For example, consider the plant $k_{l}=12.65, k_{2}=1.92$, the sampling period $\mathrm{h}_{1}=0.02$ and the controller $L_{l}=\left[\begin{array}{ll}-121 & -6.5\end{array}\right]$

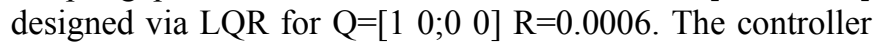
parameters $L_{\alpha}$ are continuous functions of $\alpha$, as we can see in Fig. 2 a) and b).
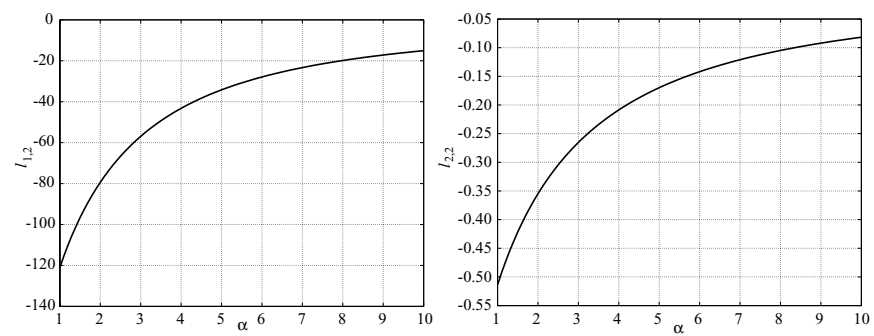

Figure 2: Controller parameters as function of $\alpha$.

\subsection{Upper diagonal matrices}

Here, we solve a numerical problem using the Case 2. A paper machine head box model taken from (Franklin et al., 1997) is as follows:

$\dot{x}=\left[\begin{array}{ccc}-0.2 & 0.1 & 1 \\ -0.05 & 0 & 0 \\ 0 & 0 & -1\end{array}\right] x+\left[\begin{array}{cc}0 & 1 \\ 0 & 0.7 \\ 1 & 0\end{array}\right]\left[\begin{array}{ll}u_{1} & u_{2}\end{array}\right]$
This matrix can be brought to diagonal form (different eigenvalues) and is also stable. The similarity transformation (12) $R$ is

$$
R=\left[\begin{array}{ccc}
-1.24223 & -.02133 & 1.4556 \\
-0.06211 & -0.3642 & 0.4263 \\
1 & 0 & 0
\end{array}\right], e^{T t}=\left[\begin{array}{ccc}
e^{-0.17071 t} & 0 & 0 \\
0 & e^{-0.0292 t} & 0 \\
0 & 0 & e^{-t}
\end{array}\right]
$$

The gain matrix of the state-feedback controller is

$$
L_{t}=\left[\begin{array}{lll}
l_{t, 1} & l_{t, 2} & l_{t, 3} \\
l_{t, 4} & l_{t, 5} & l_{t, 6}
\end{array}\right]
$$

The transformation of matrix $\left(\mathrm{R}^{-1} \mathrm{~B} \mathrm{~L}_{t} \mathrm{R}\right)$ into an upper diagonal matrix requires to adopt the solution $l_{t, 1}=0, l_{t, 2}=0, l_{t, 5}=-3.4142 l_{t, 4}$.

For $\mathrm{h}=0.2$ results

$\left(\mathrm{R}^{-1} \mathrm{~B} \mathrm{~L}_{1} \mathrm{R}\right)=\left[\begin{array}{ccc}0 & 0.504 l_{t, 4} & -0.504 l_{t, 4}+l_{t, 3}+0.489 l_{t, 6} \\ 0 & -1.39 l_{t, 4} & 1.39 l_{t, 4}+l_{t, 3}-1.35 l_{t, 6} \\ 0 & 0 & l_{t, 3}\end{array}\right]$

We can see that the first column elements cannot be modified by changing the controller parameters. Thus, $\Phi_{t,(1,1)}^{C L}$ remains unchanged (equals its original value).

Using this controller, the closed-loop matrix (for $h_{l}=0.2$ ) results

$$
\Phi_{h_{1}}^{C L}=\left[\begin{array}{ccc}
0.966 & 0.099 l_{1,4} & -0.099 l_{1,4}+0.196 l_{1,3}+0.096 l_{1,6} \\
0 & 0.994-0.277 l_{1,4} & 0.277 l_{1,4}+0.194 l_{1,3}-0.269 l_{1,6} \\
0 & 0 & 0.8187+0.181 l_{1,3}
\end{array}\right]
$$

Its eigenvalues are $\left\{0.966,0.994-0.277 l_{1,4}, 0.8187+0.181 l_{1,3}\right\}$ Thus, we have a fixed eigenvalue at 0.966 and the others two can be placed by using $l_{l, 3}$ and $l_{l, 4}$.

In the same way for $h_{2}=0.4$

$\Phi_{h 2}^{C L}=\left[\begin{array}{ccc}0.934 & 0.195 l_{2,4} & -0.195 l_{1,4}+0.386 l_{2,3}+0.189 l_{2,6} \\ 0 & 0.988-0.552 l_{2,4} & 0.553 l_{2,4}+0.397 l_{2,3}-0.536 l_{2,6} \\ 0 & 0 & 0.6703+0.3296 l_{2,3}\end{array}\right]$

Its eigenvalues are $\left\{0.934,0.988-0.552 l_{2,4}, 0.6703+0.3296 l_{2,3}\right\}$

Thus, we have a fixed eigenvalue at 0.934 and the others two can be placed by using $l_{2,4} \mathrm{y} f_{2,3}$.

In the same way we can proceed for any sampling period.

As each closed-loop matrix has an upper diagonal form, the generated algebra associated with a family of them is solvable. The initial introduction of the similarity matrix transformation $\left(\mathrm{R}\right.$ and $\left.\mathrm{R}^{-1}\right)$ is a key feature that makes this controller design possible.

\section{CONCLUSIONS}

Rate adaptation of task execution is increasingly used in order to optimize allocation and throughput of shared resources in communication and computing systems. This practice may provoke significant variations in the sampling time of digital controllers immersed in networked control systems, with negative impact in closed-loop stability and 
performance. Motivated by these problems, this paper addressed the problem of designing a controller family that, given a continuous-time linear plant sampled at a varying rate, asymptotically stabilizes the closed loop.

The solvability of the Lie algebra generated by a family of stable matrices, as a sufficient condition for the asymptotic stability of the discrete-time switching system represented by them, is a previous result being used to solve the problem. This condition has been imposed to the closed-loop sampled system in order to obtain the parameterisation of a given state-feedback controller structure that solves the problem. Conditions to find the solutions have been provided for three cases, which have been illustrated with examples.

In a paper following this one, this work has been complemented with some results on closed-loop performance and robustness, that we have obtained via the calculation of the joint spectral radius of the set of matrices defining the discrete-time switching system.

In what concerns future work, we will explore other Liealgebra solvability conditions, essentially in order to be able to take into account different types of controller structures, beyond the state-feedback controllers considered in this research.

Acknowledgments: the authors are grateful to the unknown reviewers whose suggestions helped improving the clarity of the presentation.

\section{REFERENCES}

Antunes A., Pedreiras P., Almeida L., Mota A. (2007), Dynamic Rate and Control Adaptation in Networked Control Systems, $5^{\text {th }}$ Intern. Conf. Indin, 2: 841-847.

Blondel V., Theys J., Nesterov Y. (2004). Approximations of the rate of growth of switched linear systems. Lecture Notes in Comp. Science. Conf. Hybrid Systems: Computation and Control: 7th Int. Workshop, HSCC USA, p.173-186. Springer-Verlag.

Cervin, A., Eker, J., Bernhardsson. B., and Årzén, K.-E. (2002). Feedback feedforward scheduling of control tasks. Real-Time Systems, 23(1-2), pp. 25-53.

Eker, J., Hagander, P., and Årzén, K.E. (2000), A Feedback Scheduler for Real-Time Controller Tasks. Control Engineering Practice, 12(8), p. 1369-1378.

Felicioni, F. and Junco S. (2007). Control de Tráfico de Redes de Comunicación de Sistemas Distribuidos de Control, XII RPIC, Argentina.

Franklin G., Powell D., and Workman M. (1997) Digital Control of Dynamic Systems, $3^{\text {rd }}$ edition AddisonWesley.

Gurvits L. (1995) Stability of discrete linear inclusion. Linear Algebra and its Applications, 231:47, 85.
Hespanha J. and Morse S. (1999) Input-output gains of switched linear systems. Open Problems in Mathematical Systems Theory and Control, V. Blondel, E. Sontag, M. Vidyasagar, and J. Willems: SpringerVerlag.

Liberzon D. and Morse S. (1999) Basic problems in stability and design of switched systems. IEEE Control Systems Magazine, October 1999.

Kumpati S. Narendra and Balakrishnan J. (1994), A Common Lyapunov Function for Stable LTI Systems with Commuting A-Matrices. IEEE Transactions on Automatic Control, 39(12).

Sala A. (2005) Computer control under time-varying sampling period: An LMI gridding approach. Automatica. 41(12): 2019-2192.

Schinkel M., W.-H Chen and Rantzer A. (2002). Optimal control for systems with varying sampling rate. In proceedings of American Control Conference.

Theys J. (2005) Joint Spectral Radius: theory and approximations, $\mathrm{PhD}$ Thesys. Universite catholique de Louvain Faculte des sciences appliqués Departement d'ingenierie mathematique. Center for Systems Engineering and Applied Mechanics.

Velasco M., J. M. Fuertes, P. Marti and Brandt S. A. (2004), A Control Approach to Bandwidth Management in Networked Control Systems, Proc. 30 ${ }^{\text {th }}$ IEEE IECON, Korea.

\section{Appendix A. FIRST APPENDIX}

In order to verify conditions (11) for the plant (16), the controller parameters $L_{\alpha}=\left[\begin{array}{ll}l_{1, \alpha} & l_{2, \alpha}\end{array}\right]$ for the sampling period $\alpha h$ were obtained in a symbolic form by using MAPLE, as follow:

$$
\begin{aligned}
& l_{\alpha, 1}=\left(-l_{1,1} k_{1}^{2} \alpha e^{-k_{1} \alpha h_{1}}+e^{-k_{1} h_{1}}-1-e^{-k_{1} h_{1}(\alpha+1)}\right) /\left(-h_{1} k_{1} k_{2} \alpha e^{-k_{1} \alpha h_{1}} l_{1,1}\right. \\
& +h_{1} k_{1} k_{2} \alpha l_{1,1}-h_{1} k_{1} k_{2} \alpha^{2} l_{1,1}+h_{1} k_{1} k_{2} \alpha^{2} e^{-k_{1} h_{1}} l_{1,1}+2 k_{2} e^{-k_{1} \alpha h_{1}} l_{1,1} \\
& -k_{2} e^{-2 k_{1} \alpha h_{1}} l_{1,1}-k_{2} l_{1,1}+\alpha k_{2} l_{1,1} k_{2} \alpha e^{-k_{1} \alpha h_{1}} l_{1,1}+k_{2} \alpha e^{-k_{1} h_{1}(\alpha+1)} l_{1,1} \\
& -k_{2} \alpha e^{-k_{1} h_{1}} l_{1,1}+k_{1} k_{2} e^{-2 k_{1} \alpha h_{1}} l_{1,2}+k_{1} k_{2} l_{1,2}+k_{1} k_{2} \alpha e^{-k_{1} \alpha h_{1}} l_{1,2} \\
& -k_{1} k_{2} \alpha e^{-k_{1} h_{1}(\alpha+1)} l_{1,2}-2 k_{1} k_{2} \alpha e^{-k_{1} \alpha h_{1}} l_{1,2}+k_{1} k_{2} \alpha e^{-k_{1} h_{1}} l_{1,2} \\
& \left.-k_{1} k_{2} \alpha l_{1,2}-k_{1}^{2} \alpha e^{-k_{1} \alpha h_{1}}+k_{1}^{2} \alpha-k_{1}^{2} \alpha e^{-k_{1} h_{1}}+k_{1}^{2} \alpha e^{-k_{1}(\alpha+1) h_{1}}\right) \\
& l_{\alpha, 2}=-k_{1}\left(e^{-2 k_{1} \alpha h_{1}} l_{1,1}-k_{1} e^{-2 k_{1} \alpha h_{1}} l_{1,2}-\alpha e^{-k_{1}(\alpha+1) h_{1}} l_{1,1}+\alpha e^{-k_{1} \alpha h_{1}} l_{1,1}\right. \\
& \left.-2 e^{-k_{1} \alpha h_{1}} l_{1,1}+2 k_{1} e^{-k_{1} \alpha h_{1}} l_{1,2}+\alpha e^{-k_{1} h_{1}} l_{1,1}-k_{1} l_{1,2}-\alpha l_{1,1}+l_{1,1}\right) / \\
& \left(-k_{1} k_{2} h_{1} \alpha e^{-k_{1} \alpha h_{1}} l_{1,1}+k_{1} k_{2} h_{1} \alpha l_{1,1}-k_{1} k_{2} h_{1} \alpha^{2} l_{1,1}+k_{1} k_{2} h_{1} \alpha^{2} e^{-k_{1} h_{1}} l_{1,1}\right. \\
& +2 k_{2} e^{-k_{1} \alpha h_{1}} l_{1,1}+k_{2} e^{-k_{1} \alpha h_{1}} l_{1,1}-k_{2} l_{1,1}+k_{2} \alpha l_{1,1}-k_{2} \alpha e^{-k_{1} \alpha h_{1}} l_{1,1} \\
& +k_{2} \alpha e^{-k_{1}(\alpha+1) h_{1}} l_{1,1}-k_{2} \alpha e^{-k_{1} h_{1}} l_{1,1}+k_{1} k_{2} e^{-k_{1} \alpha h_{1}} l_{1,2}+k_{1} k_{2} l_{1,2} \\
& +k_{1} k_{2} \alpha e^{-k_{1} \alpha h_{1}} l_{1,2}-k_{1} k_{2} \alpha e^{-k_{1}(\alpha+1) h_{1}} l_{1,2}-2 k_{1} k_{2} e^{-k_{1} \alpha h_{1}} l_{1,2}+k_{1} k_{2} e^{-k_{1} h_{1}} l_{1,2} \\
& \left.-k_{1} k_{2} l_{1,2}-k_{1}^{2} \alpha e^{-k_{1} \alpha h_{1}}+k_{1}^{2} \alpha-k_{1}^{2} \alpha e^{-k_{1} h_{1}}+k_{1}^{2} \alpha e^{-k_{1}(\alpha+1) h_{1}}\right)
\end{aligned}
$$

